\title{
Best proximity points of $\alpha-\beta-\psi$-proximal contractive mappings in partially ordered complete metric spaces
}

\author{
Gutti Venkata Ravindranadh Babu, Leta Bekere Kumssa \\ and Pathina Sudheer Kumar
}

\begin{abstract}
In this paper, we define $\alpha-\beta-\psi-$ proximal contractive mappings in partially ordered metric spaces and prove the existence of best proximity points of these maps in partially ordered complete metric spaces. These results extend/generalize the results of Asgari and Badehian, J. Nonl. Sci. and Appl., 2015. We provide illustrative examples in support of our theorems.
\end{abstract}

\section{INTRODUCTION}

In recent research in the field of nonlinear functional analysis, many researchers are interested in dealing with non-selfmaps to determine the distance between two nonempty subsets of a metric space $(X, d)$. Let $A$ and $B$ be two nonempty subsets of a metric space $(X, d)$ and $T: A \rightarrow B$ be a nonselfmapping. Then $d(x, T x) \geq d(A, B)$, for all $x \in A$, where $d(A, B)=$ $\inf \{d(x, y): x \in A, y \in B\}$. In general, for a non-selfmapping $T: A \rightarrow B$, the fixed point equation $T x=x$ may not have a solution. In such cases, one intend to find an approximate solution $x \in A$ such that $d(x, T x)=d(A, B)$. Best approximation theorems and best proximity point theorems are relevant in this regard. For instance, let us consider the well known classical best approximation theorem by Ky Fan [12].

Theorem 1.1 ([12]). Let $A$ be a non-empty compact convex subset of a normed linear space $X$ and $T: A \rightarrow X$ be a continuous function. Then there exists $x \in A$ such that

$$
\|x-T x\|=d(T x, A)=\inf \{\|T x-a\|: a \in A\} .
$$

2010 Mathematics Subject Classification. Primary: 47H10; Secondary: 54H25.

Key words and phrases. Best proximity point, proximal contractive maps, admissible map, partially ordered complete metric space.

Full paper. Received 21 December 2017, revised 26 January 2018, accepted 16 April 2018, available online 25 June 2018. 
On the other hand, though best approximation theorems only ensure the existence of approximate solutions, in this case, such results need not yield optimal solutions. But, best proximity point theorems provide sufficient conditions that assure the existence of approximate solutions which are optimal. In this regard, the best proximity point evolves as a generalization of the best approximation. The authors Basha [5], Choudhury, Maity and Konar, [9, 10] and Kutbi, Chandok and Sintunavarat [16] tried to reduce the problem of finding approximate solutions to that of finding optimal approximate solutions.

In recent years, the existence of best proximity points is an interesting aspect of optimization theory which attracted the attention of many researchers. For example, Abkar and Gableh [2], Basha [6], Caballaro, Harjani and Sadarangani [7], Eldred [11], Gabeleh [13] and Karapinar [15] and the related references cited in these papers, worked in this area.

A best proximity point becomes a fixed point if the underlying mapping is a selfmapping. Therefore, it is concluded that best proximity point theorems generalize fixed point theorems in a natural way. For more works on the existence of best proximity points, we refer $[1,3,8,14,17]$ and references therein.

Our purpose here is to establish best proximity point theorems in partially ordered metric spaces.

We recall the following notation and definitions. Let $(X, d, \preceq)$ be a partially ordered metric space and let $A$ and $B$ be nonempty subsets of $X$.

$$
\begin{aligned}
& A_{0}:=\{x \in A: d(x, y)=d(A, B) \text { for some } y \in B\}, \\
& B_{0}:=\{y \in B: d(x, y)=d(A, B) \text { for some } x \in A\} .
\end{aligned}
$$

Definition 1.1 ([6]). A mapping $T: A \rightarrow B$ is said to be proximally increasing if for all $u_{1}, u_{2}, x_{1}, x_{2} \in A, x_{1} \preceq x_{2}$ and $d\left(u_{1}, T x_{1}\right)=d\left(u_{2}, T x_{2}\right)=$ $d(A, B)$ imply $u_{1} \preceq u_{2}$.

We denote by $\Psi$ a family of functions $\psi:[0, \infty) \rightarrow[0, \infty)$ such that for each $\psi \in \Psi$ and $t>0$,

(i) $\psi$ is nondecreasing,

(ii) $\sum_{n=1}^{\infty} \psi^{n}(t)<+\infty$, where $\psi^{n}$ is the $n$-th iterate of $\psi$.

Remark 1.1. If $\psi \in \Psi$, then $\psi^{n}(t) \rightarrow 0$ as $n \rightarrow \infty$, for all $t \geq 0$ and $\psi(t)<t$, for all $t>0$.

Definition $1.2([4])$. Let $(X, \preceq)$ be a partially ordered space with metric $d$. We say that $f: X \rightarrow X$ is an $\alpha-\beta-\psi$-contractive mapping if there exist three functions $\alpha, \beta: X \times X \rightarrow[0, \infty), \psi \in \Psi$ such that

$$
\alpha(x, y) d(f(x), f(y)) \leq \beta(x, y) \psi(d(x, y)),
$$

for all $x, y \in X$ with $x \succeq y$. 
Definition 1.3 ([4]). Let $f: X \rightarrow X, \alpha, \beta: X \times X \rightarrow[0, \infty)$ and $C_{\alpha}>$ $0, C_{\beta} \geq 0$. We say that $f$ is an $\alpha-\beta$-admissible mapping, if for all $x, y \in X$ with $x \succeq y$ hold

(i) $\alpha(x, y) \geq C_{\alpha} \Longrightarrow \alpha(f x, f y) \geq C_{\alpha}$,

(ii) $\beta(x, y) \leq C_{\beta} \Longrightarrow \beta(f x, f y) \leq C_{\beta}$,

(iii) $0 \leq \frac{C_{\beta}}{C_{\alpha}} \leq 1$.

In 2015, Asgari and Badehian [4], proved fixed point theorems for $\alpha-\beta-$ $\psi$-contractive mappings in partially ordered space with complete metric.

Theorem $1.2([4])$. Let $(X, \preceq)$ be a partially ordered space with complete metric $d$. Let $f: X \rightarrow X$ be a nondecreasing, $\alpha-\beta-\psi$-contractive mapping satisfying the following conditions:

(i) $f$ is continuous,

(ii) $f$ is $\alpha-\beta$-admissible,

(iii) there exists $x_{0} \in X$ such that $x_{0} \preceq f x_{0}$,

(iv) there exist $C_{\alpha}>0, C_{\beta} \geq 0$ such that $\alpha\left(f x_{0}, x_{0}\right) \geq C_{\alpha}, \beta\left(f x_{0}, x_{0}\right) \leq$ $C_{\beta}$.

Then, $f$ has a fixed point.

In Section 2, we introduce a notion of $\alpha-\beta$-proximal admissible mappings and $\alpha-\beta-\psi-$ proximal contractive mappings that we consider to prove our main results in Section 3. In Section 4, we draw some corollaries and provide examples in support of our main results.

\section{Preliminaries}

Definition 2.1. Let $(X, d, \preceq)$ be a partially ordered metric space, $A, B$ be two nonempty subsets of $X, \alpha, \beta: A \times A \rightarrow[0, \infty)$ be functions, $C_{\alpha}>$ $0, C_{\beta} \geq 0$ be two constants and $T: A \rightarrow B$ be a non-selfmapping. We say that $T$ is an $\alpha-\beta$-proximal admissible, if for all $x, y, u, v \in A$, with $x \preceq y$ hold

(i) $\alpha(x, y) \geq C_{\alpha}$ and $d(u, T x)=d(v, T y)=d(A, B)$ implies $\alpha(u, v) \geq C_{\alpha}$,

(ii) $\beta(x, y) \leq C_{\beta}$ and $d(u, T x)=d(v, T y)=d(A, B)$ implies $\beta(u, v) \leq C_{\beta}$,

(iii) $0 \leq \frac{C_{\beta}}{C_{\alpha}} \leq 1$.

If (i), (ii) and (iii) hold for $\alpha(x, y)=1=\beta(x, y)$, for all $x, y \in A$, then we say that $T$ is proximal admissible.

Remark 2.1. If $A=B=X$ in Definition 2.1, then $T$ is an $\alpha$ - $\beta$-admissible mapping.

Example 2.1. Let $X=[0, \infty) \times[0, \infty)$ with the Euclidean metric $d$. Let $A=\{1\} \times[0, \infty), B=\{2\} \times[0, \infty)$. We define a partial order $\preceq$ on $X$ by $(x, y) \preceq(u, v)$ if and only if $x \leq u$ and $y \leq v$, for all $(x, y),(u, v) \in X$, where $\preceq$ is the usual order on $[0, \infty)$. We define $T: A \rightarrow B$ by

$$
T(1, x)=\left(2,2^{x}\right), \quad \text { if } x \in[0, \infty) .
$$


We also define functions $\alpha, \beta: A \times A \rightarrow[0, \infty)$ by

$$
\begin{aligned}
& \alpha((1, x),(1, y))= \begin{cases}\frac{3}{2}, & \text { if }(1, x) \preceq(1, y), \\
0, & \text { otherwise. }\end{cases} \\
& \beta((1, x),(1, y))= \begin{cases}\frac{1}{2}, & \text { if }(1, x) \preceq(1, y), \\
0, & \text { otherwise. }\end{cases}
\end{aligned}
$$

We choose $C_{\alpha}=1$ and $C_{\beta}=\frac{2}{3}$. Clearly, $0 \leq \frac{C_{\beta}}{C_{\alpha}} \leq 1$.

Let $(1, x),(1, y),(1, u)$ and $(1, v) \in A$, such that

(i) $\alpha((1, x),(1, y))=\frac{3}{2} \geq 1=C_{\alpha}$ and $d((1, u), T(1, x))=d((1, v), T(1, y))=d(A, B)=1$,

(ii) $\beta((1, x),(1, y))=\frac{1}{2} \leq \frac{2}{3}=C_{\beta}$ and $d((1, u), T(1, x))=d((1, v), T(1, y))=d(A, B)=1$.

From (i) and (ii), we obtain $u=2^{x}$ and $v=2^{y}$.

Since $(1, x) \preceq(1, y)$, it follows that $(1, u) \preceq(1, v)$, so $\alpha((1, u),(1, v))=$ $\frac{3}{2} \geq 1=C_{\alpha}$ and $\beta((1, u),(1, v))=\frac{1}{2} \leq \frac{2}{3}=\bar{C}_{\beta}$. Hence $T$ is $\alpha-\beta$-proximal admissible.

Definition 2.2. Let $(X, d, \preceq)$ be a partially ordered metric space and $A$ and $B$ be nonempty closed subsets of $X$. We say that $T: A \rightarrow B$ is an $\alpha-\beta-\psi$-proximal contractive mapping if there exist functions $\alpha, \beta$ : $A \times A \rightarrow[0, \infty), \psi \in \Psi$ such that for all $x, y, u, v \in A$ with $x \preceq y$ holds:

$$
\left.\begin{array}{l}
d(u, T x)=d(A, B) \\
d(v, T y)=d(A, B)
\end{array}\right\} \Longrightarrow \alpha(x, y) d(u, v) \leq \beta(x, y) \psi(d(x, y)) \text {. }
$$

If $\alpha(x, y)=1=\beta(x, y)$, for all $x, y \in A$ in (1) then we say that $T$ is a $\psi$-proximal contractive mappings.

Remark 2.2. Here we observe that if $A=B=X$ in Definition 2.2, then $T$ is an $\alpha-\beta-\psi$-contractive mapping.

Example 2.2. Let $A=\{0\} \times[0, \infty), B=\{-1\} \times[0, \infty)$ and $X=A \cup B$, with the Euclidean metric $d$. We define a partial order $\preceq$ on $X$ by $(x, y) \preceq(u, v)$ if and only if $x \leq u$ and $y \leq v$, for all $(x, y),(u, v) \in X$.

Clearly, holds $d(A, B)=1$. We define $T: A \rightarrow B$ by

$$
T(0, x)= \begin{cases}\left(-1, \frac{x^{2}}{1+x}\right), & \text { if } x \in[0,1], \\ \left(-1,2 x-\frac{3}{2}\right), & \text { if } x \in[1, \infty) .\end{cases}
$$

We also define functions $\alpha, \beta: A \times A \rightarrow[0, \infty)$ by

$$
\begin{aligned}
& \alpha((0, x),(0, y))= \begin{cases}\frac{3}{4} & \text { if } x, y \in[0,1], \text { with }(0, x) \preceq(0, y), \\
0 & \text { otherwise. }\end{cases} \\
& \beta((0, x),(0, y))= \begin{cases}\frac{2}{3} & \text { if } x, y \in[0,1], \text { with }(0, x) \preceq(0, y), \\
0 & \text { otherwise. }\end{cases}
\end{aligned}
$$


Let $\psi(t)=\frac{7}{8} t$, for all $t \geq 0$. We will show that $T$ is an $\alpha-\beta-\psi$-proximal contractive mapping.

Let $(0, x),(0, y),(0, u)$ and $(0, v) \in A$ with $(0, x) \preceq(0, y)$ such that

$$
d((0, u), T(0, x))=d((0, v), T(0, y))=d(A, B)=1 .
$$

If $x, y \in[0,1]$, with $(0, x) \preceq(0, y)$, then $\alpha((0, x),(0, y))=\frac{3}{4}$ and $\beta((0, x)$, $(0, y))=\frac{2}{3}$.

From (2), we obtain $u=\frac{x^{2}}{1+x} \in\left[0, \frac{1}{2}\right]$ and $v=\frac{y^{2}}{1+y} \in\left[0, \frac{1}{2}\right]$.

Therefore,

$$
\begin{aligned}
& \alpha((0, x),(0, y)) d((0, u),(0, v))=\frac{3}{4}|u-v| \\
= & \frac{3}{4}\left|\frac{x^{2}}{1+x}-\frac{y^{2}}{1+y}\right|=\frac{3}{4}\left(\frac{|x-y|(x+y+x y)}{(1+x)(1+y)}\right) \\
\leq & \frac{7}{12}|x-y|=\beta((0, x),(0, y)) \psi(d((0, x),(0, y))) .
\end{aligned}
$$

For the other possible cases, the inequality (1) holds trivially. Hence $T$ is an $\alpha-\beta-\psi$-proximal contractive mapping.

Here we observe that the inequality (1) fails to hold for any $\psi \in \Psi$, if $\alpha((0, x),(0, y))=\beta((0, x),(0, y))=1$ such that $x, y \in[1, \infty)$ with $(0, x) \preceq$ $(0, y)$ and $x \neq y$. Let $(0, x),(0, y),(0, u)$ and $(0, v) \in A$, with $(0, x) \preceq(0, y)$ and $x, y \in[1, \infty)$, such that

$$
d((0, u), T(0, x))=d((0, v), T(0, y))=d(A, B)=1 .
$$

From (3), we get $u=2 x-\frac{3}{2}$ and $v=2 y-\frac{3}{2}$.

Therefore,

$$
d((0, u),(0, y))=2|x-y| \not \leq \psi(|x-y|)=\psi(d((0, x),(0, y))) .
$$

This shows the importance of $\alpha$ and $\beta$ in the inequality (1).

In the following, we prove our main results.

\section{Main Results}

Theorem 3.1. Let $(X, d, \preceq)$ be a partially ordered complete metric space. Let $A, B$ be non-empty closed subsets of $X$ with $A_{0}$ is nonempty and closed. Let $T: A \rightarrow B$ be a proximally increasing non-selfmapping such that the following conditions hold:

(i) $T$ is continuous,

(ii) $T$ is an $\alpha-\beta-\psi$-proximal contractive mapping,

(iii) $T$ is an $\alpha-\beta$-proximal admissible,

(iv) $T\left(A_{0}\right) \subseteq B_{0}$,

(v) there exist elements $x_{0}, x_{1} \in A_{0}$ such that $x_{0} \preceq x_{1}$ and $d\left(x_{1}, T x_{0}\right)=$ $d(A, B)$,

(vi) there exist $C_{\alpha}>0, C_{\beta} \geq 0$ such that $\alpha\left(x_{0}, x_{1}\right) \geq C_{\alpha}, \beta\left(x_{0}, x_{1}\right) \leq C_{\beta}$. 
Then $T$ has a best proximity point in $A_{0}$.

Proof. By condition $(v)$, there exist $x_{0}, x_{1} \in A_{0}$ such that $x_{0} \preceq x_{1}$ and

$$
d\left(x_{1}, T x_{0}\right)=d(A, B) .
$$

Since $T\left(A_{0}\right) \subseteq B_{0}$, we have $T x_{1} \in B_{0}$ and hence there exists an element $x_{2} \in A$ such that

$$
d\left(x_{2}, T x_{1}\right)=d(A, B) .
$$

Since $T$ is proximally increasing on $A$, from (4) and (5), we have $x_{1} \preceq x_{2}$. On continuing this process, we get a sequence $\left\{x_{n}\right\}$ in $A_{0}$ such that

$$
\left.\begin{array}{l}
d\left(x_{n}, T x_{n-1}\right)=d(A, B) \\
d\left(x_{n+1}, T x_{n}\right)=d(A, B)
\end{array}\right\}
$$

satisfying

$$
x_{1} \preceq x_{2} \preceq x_{3} \preceq \cdots \preceq x_{n} \preceq x_{n+1} \preceq \cdots, \quad \text { for } n=1,2,3, \ldots
$$

If $x_{n_{0}}=x_{n_{0}+1}$ for some $n_{0} \in \mathbb{N}$, then $x_{n_{0}}$ is the best proximity point of $T$ and hence the conclusion of the theorem follows.

Now, we assume with out loss of generality that any two consecutive elements of $\left\{x_{n}\right\}$ are distinct.

From condition (iii), condition (vi) and (6), the following holds:

$$
\begin{gathered}
\alpha\left(x_{0}, x_{1}\right) \geq C_{\alpha} \text { and } \beta\left(x_{0}, x_{1}\right) \leq C_{\beta} \\
d\left(x_{1}, T x_{0}\right)=d(A, B) \\
d\left(x_{2}, T x_{1}\right)=d(A, B) \\
\alpha\left(x_{1}, x_{2}\right) \geq C_{\alpha} \text { and } \beta\left(x_{1}, x_{2}\right) \leq C_{\beta} .
\end{gathered} \Longrightarrow
$$

Since $T$ is an $\alpha-\beta-\psi$-proximal contractive mapping and by considering (7), we have

$$
\begin{aligned}
C_{\alpha} d\left(x_{1}, x_{2}\right) & \leq \alpha\left(x_{0}, x_{1}\right) d\left(x_{1}, x_{2}\right) \\
& \leq \beta\left(x_{0}, x_{1}\right) \psi\left(d\left(x_{0}, x_{1}\right)\right) \\
& \leq C_{\beta} \psi\left(d\left(x_{0}, x_{1}\right)\right),
\end{aligned}
$$

therefore

$$
d\left(x_{1}, x_{2}\right) \leq \frac{C_{\beta}}{C_{\alpha}} \psi\left(d\left(x_{0}, x_{1}\right) \leq \psi\left(d\left(x_{0}, x_{1}\right) .\right.\right.
$$

Again by condition (iii), condition (vi), (6) and (7), we have

$$
\begin{gathered}
\alpha\left(x_{1}, x_{2}\right) \geq C_{\alpha}, \beta\left(x_{1}, x_{2}\right) \leq C_{\beta} \\
d\left(x_{2}, T x_{1}\right)=d(A, B) \\
d\left(x_{3}, T x_{2}\right)=d(A, B) \\
\alpha\left(x_{2}, x_{3}\right) \geq C_{\alpha} \text { and } \beta\left(x_{2}, x_{3}\right) \leq C_{\beta} .
\end{gathered}
$$


Therefore, by considering (9) and by the fact that $T$ is an $\alpha-\beta-\psi-$ proximal contractive mapping, we have

$$
\begin{aligned}
C_{\alpha} d\left(x_{2}, x_{3}\right) & \leq \alpha\left(x_{1}, x_{2}\right) d\left(x_{2}, x_{3}\right) \\
& \leq \beta\left(x_{1}, x_{2}\right) \psi\left(d\left(x_{1}, x_{2}\right)\right) \\
& \leq C_{\beta} \psi\left(d\left(x_{1}, x_{2}\right)\right),
\end{aligned}
$$

and hence

$$
d\left(x_{2}, x_{3}\right) \leq \frac{C_{\beta}}{C_{\alpha}} \psi\left(d\left(x_{1}, x_{2}\right) \leq \psi\left(d\left(x_{1}, x_{2}\right) \leq \psi^{2}\left(d\left(x_{0}, x_{1}\right)\right) .\right.\right.
$$

On continuing this process, we obtain

$$
\begin{gathered}
\alpha\left(x_{n-1}, x_{n}\right) \geq C_{\alpha}, \beta\left(x_{n-1}, x_{n}\right) \leq C_{\beta} \\
d\left(x_{n}, T x_{n-1}\right)=d(A, B) \\
d\left(x_{n+1}, T x_{n}\right)=d(A, B) \\
\alpha\left(x_{n}, x_{n+1}\right) \geq C_{\alpha} \text { and } \beta\left(x_{n}, x_{n+1}\right) \leq C_{\beta},
\end{gathered}
$$

for $n=1,2,3, \ldots$ and

$$
d\left(x_{n}, x_{n+1}\right) \leq \psi^{n}\left(d\left(x_{0}, x_{1}\right)\right) .
$$

Since $\psi \in \Psi$, we have $\psi^{n}\left(d\left(x_{0}, x_{1}\right)\right) \rightarrow 0$ as $n \rightarrow \infty$.

Now, we show that $\left\{x_{n}\right\}$ is a Cauchy sequence. We fix $\epsilon>0$ and choose $n_{0} \in \mathbb{N}$ such that $\sum_{n=n_{0}}^{\infty} \psi^{n}\left(d\left(x_{0}, x_{1}\right)\right)<\epsilon$. Let $m, n \in \mathbb{N}$ with $m>n>n_{0}$. Therefore by applying triangle inequality, we have

$$
\begin{aligned}
d\left(x_{n}, x_{m}\right) & \leq d\left(x_{n}, x_{n+1}\right)+d\left(x_{n+1}, x_{n+2}\right)+\ldots+d\left(x_{m-1}, x_{m}\right) \\
& \leq \psi^{n}\left(d\left(x_{0}, x_{1}\right)\right)+\psi^{n+1}\left(d\left(x_{0}, x_{1}\right)\right)+\ldots+\psi^{m-1}\left(d\left(x_{0}, x_{1}\right)\right) \\
& =\sum_{n=n_{0}}^{m-1} \psi^{n}\left(d\left(x_{0}, x_{1}\right)\right) \leq \sum_{n=n_{0}}^{\infty} \psi^{n}\left(d\left(x_{0}, x_{1}\right)\right)<\epsilon .
\end{aligned}
$$

Hence $\left\{x_{n}\right\}$ is a Cauchy sequence. Since $A_{0}$ is a closed subset of a complete metric space and hence it is complete, there exists $x \in A_{0}$ such that $x_{n} \rightarrow x$. Since $T$ is continuous, by letting $n \rightarrow \infty$ in (6), we obtain $d(x, T x)=$ $d(A, B)$. Hence $x$ is a best proximity point of $T$.

If we drop the continuity assumption from Theorem 3.1, we obtain the following result.

Theorem 3.2. Let $(X, d, \preceq)$ be a partially ordered complete metric space. Let $A, B$ be non-empty closed subsets of $X$ with $A_{0}$ is nonempty and closed. Let $T: A \rightarrow B$ be proximally increasing non-selfmapping such that the following conditions hold:

(i) $T$ is an $\alpha-\beta-\psi$-proximal contractive mapping and $T$ is an $\alpha-\beta-$ proximal admissible; 
(ii) $T\left(A_{0}\right) \subseteq B_{0}$;

(iii) there exist elements $x_{0}, x_{1} \in A_{0}$ such that $x_{0} \preceq x_{1}$ and $d\left(x_{1}, T x_{0}\right)=$ $d(A, B)$;

(iv) there exist $C_{\alpha}>0, C_{\beta} \geq 0$ such that $\alpha\left(x_{0}, x_{1}\right) \geq C_{\alpha}, \beta\left(x_{0}, x_{1}\right) \leq C_{\beta}$;

(v) if $\left\{x_{n}\right\}$ is a sequence in $A$ such that $\alpha\left(x_{n}, x_{n+1}\right) \geq C_{\alpha}, \beta\left(x_{n}, x_{n+1}\right) \leq$ $C_{\beta}$, for all $n \in \mathbb{N}, x_{n} \rightarrow x$ as $n \rightarrow \infty$, then $\alpha\left(x_{n}, x\right) \geq C_{\alpha}$ and $\beta\left(x_{n}, x\right) \leq C_{\beta}$;

(vi) if $\left\{x_{n}\right\}$ is a nondecreasing sequence in $A$ such that $x_{n} \rightarrow x$ as $n \rightarrow \infty$, then $x_{n} \preceq x$ for all $n \in \mathbb{N}$.

Then $T$ has a best proximity point in $A_{0}$.

Proof. From the proof of Theorem 3.1, we have the sequence $\left\{x_{n}\right\}$ is Cauchy and $x_{n} \rightarrow x \in A_{0}$. Since $T\left(A_{0}\right) \subseteq B_{0}$, then $T(x) \in B_{0}$ and hence there exists $z \in A$ such that

$$
d(z, T x)=d(A, B) .
$$

In the proof of Theorem 3.1, we obtained that $\left\{x_{n}\right\}$ is a nondecreasing sequence satisfying $\alpha\left(x_{n}, x_{n+1}\right) \geq C_{\alpha}$ and $\beta\left(x_{n}, x_{n+1}\right) \leq C_{\beta}$. Therefore, by condition $(v)$, it follows that $\alpha\left(x_{n}, x\right) \geq C_{\alpha}$ and $\beta\left(x_{n}, x\right) \leq C_{\beta}$, and condition $(v i)$, we have $x_{n} \preceq x$ for $n \in \mathbb{N}$.

We now claim that $z=x$. Since $d\left(x_{n+1}, T x_{n}\right)=d(A, B)$, by combining this equation with (10) and by the fact that $T$ is an $\alpha-\beta-\psi$-proximal contractive mapping, we have

$$
\begin{aligned}
C_{\alpha} d\left(x_{n+1}, z\right) & \leq \alpha\left(x_{n}, x\right) d\left(x_{n+1}, z\right) \\
& \leq \beta\left(x_{n}, x\right) \psi\left(d\left(x_{n}, x\right)\right) \\
& \leq C_{\beta} \psi\left(d\left(x_{n}, x\right)\right),
\end{aligned}
$$

and therefore

$$
d\left(x_{n+1}, z\right) \leq \frac{C_{\beta}}{C_{\alpha}} \psi\left(d\left(x_{n}, x\right)\right) \leq \psi\left(d\left(x_{n}, x\right)\right) .
$$

Since $\psi \in \Psi$, we get

$$
d\left(x_{n+1}, z\right)<d\left(x_{n}, x\right) .
$$

If $n \rightarrow \infty$, we obtain $x=z$. Hence $x$ is a best proximity point of $T$.

Lemma 3.1. In addition to the hypotheses of Theorem 3.1 (Theorem 3.2), if $x$ is a best proximity point of $T$ with $x \preceq u, \alpha(x, u) \geq C_{\alpha}$ and $\beta(x, u) \leq$ $C_{\beta}$ for some $u \in A_{0}$, then there exists a sequence $\left\{u_{n}\right\} \subseteq A_{0}$ such that $d\left(u_{n}, T u_{n-1}\right)=d(A, B), x \preceq u_{n}$, for $n=1,2,3, \ldots$ and $u_{n} \rightarrow x$ as $n \rightarrow \infty$.

Proof. Let $x$ be a best proximity point of $T$, i.e.,

$$
d(x, T x)=d(A, B) .
$$


Let $u \in A_{0}$ such that $x \preceq u$. We set $u_{0}=u$. Since $T\left(A_{0}\right) \subseteq B_{0}$ and $u=u_{0} \in A_{0}$, we have $T u_{0} \in B_{0}$. Hence there exists $u_{1} \in A$ such that

$$
d\left(u_{1}, T u_{0}\right)=d(A, B) .
$$

From (12), by the definition of $A_{0}$ and $B_{0}$, we have $u_{1} \in A_{0}$. Since $T$ is proximally increasing on $A_{0}$, from $x \preceq u=u_{0}$, (11) and (12), we have $x \preceq u_{1}$.

On continuing this process we can construct a sequence $\left\{u_{n}\right\}$ in $A_{0}$ such that

$$
d\left(u_{n}, T u_{n-1}\right)=d(A, B),
$$

satisfying

$$
x \preceq u_{n}, \quad \text { for } \quad n=1,2,3, \ldots
$$

By assumption, from (11) and (12) we have

$$
\left.\begin{array}{c}
\alpha\left(x, u_{0}\right) \geq C_{\alpha} \text { and } \beta\left(x, u_{0}\right) \leq C_{\beta} \\
d(x, T x)=d(A, B) \\
d\left(u_{1}, T u_{0}\right)=d(A, B) \\
\alpha\left(x, u_{1}\right) \geq C_{\alpha} \text { and } \beta\left(x, u_{1}\right) \leq C_{\beta} .
\end{array}\right\} \Longrightarrow
$$

Since $T$ is an $\alpha-\beta-\psi$-proximal contractive mapping and by considering (15), we have

$$
\begin{aligned}
C_{\alpha} d\left(x, u_{1}\right) & \leq \alpha\left(x, u_{0}\right) d\left(x, u_{1}\right) \\
& \leq \beta\left(x, u_{0}\right) \psi\left(d\left(x, u_{0}\right)\right) \\
& \leq C_{\beta} \psi\left(d\left(x, u_{0}\right)\right),
\end{aligned}
$$

and it follows that

$$
d\left(x, u_{1}\right) \leq \frac{C_{\beta}}{C_{\alpha}} \psi\left(d\left(x, u_{0}\right) \leq \psi\left(d\left(x, u_{0}\right)\right)=\psi(d(x, u)) .\right.
$$

From (11), (13), (14) and (15) we have

$$
\left.\begin{array}{c}
\alpha\left(x, u_{1}\right) \geq C_{\alpha} \text { and } \beta\left(x, u_{1}\right) \leq C_{\beta} \\
d(x, T x)=d(A, B) \\
d\left(u_{2}, T u_{1}\right)=d(A, B) \\
\alpha\left(x, u_{2}\right) \geq C_{\alpha} \text { and } \beta\left(x, u_{2}\right) \leq C_{\beta} .
\end{array}\right\} \Longrightarrow
$$

Since $T$ is an $\alpha-\beta-\psi$-proximal contractive mapping and by considering (16), we have

$$
\begin{aligned}
C_{\alpha} d\left(x, u_{2}\right) & \leq \alpha\left(x, u_{1}\right) d\left(x, u_{2}\right) \\
& \leq \beta\left(x, u_{1}\right) \psi\left(d\left(x, u_{1}\right)\right) \\
& \leq C_{\beta} \psi\left(d\left(x, u_{1}\right)\right) .
\end{aligned}
$$


Now, it follows that

$$
\begin{aligned}
d\left(x, u_{2}\right) & \leq \frac{C_{\beta}}{C_{\alpha}} \psi\left(d\left(x, u_{1}\right)\right. \\
& \leq \psi\left(d\left(x, u_{1}\right)\right) \\
& \leq \psi^{2}\left(d\left(x, u_{0}\right)\right) \\
& =\psi^{2}(d(x, u)) .
\end{aligned}
$$

On continuing this process, we obtain

$$
d\left(x, u_{n}\right) \leq \psi^{n}(d(x, u)) \rightarrow 0 \quad \text { as } \quad n \rightarrow \infty .
$$

i.e., $u_{n} \rightarrow x$ as $n \rightarrow \infty$.

Theorem 3.3. Suppose that all the hypotheses of Theorem 3.1 (Theorem 3.2) are satisfied. We Assume the following hypothesis.

Condition $(H)$ : There exists $u \in A_{0}$ such that for every $x, y \in A_{0}$ with $x \preceq u, y \preceq u$,

$$
\left\{\begin{array}{l}
\alpha(x, u) \geq C_{\alpha} \quad \text { and } \quad \beta(x, u) \leq C_{\beta}, \\
\alpha(y, u) \geq C_{\alpha} \quad \text { and } \quad \beta(y, u) \leq C_{\beta} .
\end{array}\right.
$$

Then $T$ has a unique best proximity point in $A_{0}$.

Proof. By the proof of Theorem 3.1 (Theorem 3.2), the set of best proximity points of $T$ is nonempty. Let $x, y$ be two best proximity points of $T$ in $A_{0}$. By our assumption, we have there exists $u \in A_{0}$ such that $x \preceq u, y \preceq u$, $\alpha(x, u) \geq C_{\alpha}$ and $\beta(x, u) \leq C_{\beta}$ and $\alpha(y, u) \geq C_{\alpha}$ and $\beta(y, u) \leq C_{\beta}$. Now by applying Lemma 3.1, it follows that there exists a sequence $\left\{u_{n}\right\} \subseteq A_{0}$ such that $u_{n} \rightarrow x$ and $u_{n} \rightarrow y$ as $n \rightarrow \infty$. Hence by the uniqueness of limits we have $x=y$.

\section{Corollaries and Examples}

Corollary 4.1. Let $(X, d, \preceq)$ be a partially ordered complete metric space. Let $A, B$ be non-empty closed subsets of $X$ with $A_{0}$ is nonempty and closed. Let $T: A \rightarrow B$ be proximally increasing non-selfmapping such that for all $x, y, u, v \in A$ with $x \preceq y$ hold:

(i) $d(u, T x)=d(v, T y)=d(A, B)$ implies that $d(u, v) \leq k d(x, y)$, for some $k \in[0,1)$,

(ii) $T$ is continuous and $T\left(A_{0}\right) \subseteq B_{0}$,

(iii) $T$ is $\psi$ - proximal contractive mapping and $T$ is proximal admissible,

(iv) there exist elements $x_{0}, x_{1} \in A_{0}$ such that $x_{0} \preceq x_{1}$ and $d\left(x_{1}, T x_{0}\right)=$ $d(A, B)$,

Then $T$ has a best proximity point in $A_{0}$.

Proof. Follows by choosing $\psi(t)=k t, t \geq 0$, and $\alpha(x, y)=\beta(x, y)=1$, for all $x, y$ in $A$, with $C_{\alpha}=C_{\beta}=1$ in Theorem 3.1. 
If the continuity assumption is removed from Corollary 4.1, we have the following result.

Corollary 4.2. Let $(X, d, \preceq)$ be a partially ordered complete metric space. Let $A, B$ be non-empty closed subsets of $X$ with $A_{0}$ is nonempty and closed. Let $T: A \rightarrow B$ be proximally increasing non-selfmapping such that for all $x, y, u, v \in A$ with $x \preceq y$ hold:

(i) $d(u, T x)=d(v, T y)=d(A, B)$ implies that $d(u, v) \leq k d(x, y)$, for some $k \in[0,1)$,

(ii) $T\left(A_{0}\right) \subseteq B_{0}$,

(iii) $T$ is $\psi$-proximal contractive mapping and $T$ is proximal admissible,

(iv) if $\left\{x_{n}\right\}$ is a sequence in $A$ such that $\alpha\left(x_{n}, x_{n+1}\right) \geq C_{\alpha}, \beta\left(x_{n}, x_{n+1}\right) \leq$ $C_{\beta}$ for all $n \in \mathbb{N}$ and $x_{n} \rightarrow x$ as $n \rightarrow \infty$, then $\alpha\left(x_{n}, x\right) \geq C_{\alpha}$ and $\beta\left(x_{n}, x\right) \leq C_{\beta}$,

(v) if $\left\{x_{n}\right\}$ is a nondecreasing sequence in $A$ such that $x_{n} \rightarrow x$ as $n \rightarrow \infty$, then $x_{n} \preceq x$ for all $n \in \mathbb{N}$.

Then $T$ has a best proximity point in $A_{0}$.

Proof. By choosing $\psi(t)=k t, k \in[0,1)$ and $\alpha(x, y)=\beta(x, y)=1$ for all $x, y$ in A with $C_{\alpha}=C_{\beta}=1$ in Theorem 3.2, it follows that all the hypothesis of Theorem 3.2 holds so that the conclusion of this corollary holds.

Remark 4.1. If $A=B=X$ in Theorem 3.1, then Theorem 1.2 follows as a corollary to Theorem 3.1.

The following example is in support of Theorem 3.1.

Example 4.1. Let $X=\mathbb{R}^{2}$, with an Euclidean metric $d$. We define a partial order $\preceq$ on $X$ by $(x, y) \preceq(u, v)$ if and only if $x \geq u$ and $y \geq v$, for all $(x, y),(u, v) \in X$, where $\geq$ is the usual order on $\mathbb{R}$.

Let $A=\{-1\} \times[0,4]=A_{0}, B=\{1\} \times[0,4]=B_{0}$. Clearly $d(A, B)=2$. We define $T: A \rightarrow B$ by

$$
T(-1, x)= \begin{cases}\left(1, \frac{x}{4}\right), & \text { if } x \in[0,1], \\ \left(1, \frac{5}{4} x-1\right), & \text { if } x \in[1,4]\end{cases}
$$

Clearly $T$ is continuous, proximally increasing on $A$ and $T\left(A_{0}\right) \subseteq B_{0}$.

Now, we define functions $\alpha, \beta: A \times A \rightarrow[0, \infty)$ by

$$
\begin{aligned}
& \alpha((-1, x),(-1, y))= \begin{cases}\frac{3}{4}, & \text { if } x, y \in[0,1] \text { with }(-1, x) \leq(-1, y), \\
0, & \text { otherwise. }\end{cases} \\
& \beta((-1, x),(-1, y))= \begin{cases}\frac{1}{2}, & \text { if } x, y \in[0,1] \text { with }(-1, x) \leq(-1, y), \\
0, & \text { otherwise. }\end{cases}
\end{aligned}
$$

Let $\psi(t)=\frac{t}{2}$ for all $t \geq 0$. We now show that $T$ is an $\alpha-\beta-\psi$-proximal contractive mapping. 
Now, let $(-1, x),(-1, y),(-1, u)$ and $(-1, v) \in A$ with $(-1, x) \preceq(-1, y)$ such that

$$
d((-1, u), T(-1, x))=d((-1, v), T(-1, y))=d(A, B)=2 .
$$

We consider the case for $x, y \in[0,1]$ with $(-1, x) \leq(-1, y)$.

Then $\alpha((-1, x),(-1, y))=\frac{3}{4}$ and $\beta((-1, x),(-1, y))=\frac{1}{2}$. In this case from (18), we obtain $u=\frac{x}{4}$ and $v=\frac{y}{4} \in\left[0, \frac{1}{4}\right]$. Therefore

$$
\begin{aligned}
& \alpha((-1, x),(-1, y)) d((-1, u),(-1, v)) \\
= & \frac{3}{4}|u-v| \leq|u-v|=\frac{1}{2}\left(\frac{|x-y|}{2}\right) \\
= & \beta((-1, x),(-1, y)) \psi(d((-1, x),(-1, y))) .
\end{aligned}
$$

The inequality (1) holds trivially for the other possible cases. Hence $T$ is an $\alpha-\beta-\psi$-proximal contractive mapping.

We now show that $T$ is an $\alpha-\beta$-proximal admissible. For this purpose, we choose $C_{\alpha}=\frac{2}{3}$ and $C_{\beta}=\frac{1}{2}$. Clearly $0 \leq \frac{C_{\beta}}{C_{\alpha}} \leq 1$.

Let $(-1, x),(-1, y),(-1, u)$ and $(-1, v) \in A$ with $(-1, x) \preceq(-1, y)$ with $x, y \in[0,1]$ such that:

(i) $\alpha((-1, x),(-1, y))=\frac{3}{4} \geq \frac{2}{3}=C_{\alpha}$ and

$$
d((-1, u), T(-1, x))=d((-1, v), T(-1, y))=d(A, B)=2,
$$

(ii) $\beta((-1, x),(-1, y))=\frac{1}{2} \leq \frac{1}{2}=C_{\beta}$ and

$$
d((-1, u), T(-1, x))=d((-1, v), T(-1, y))=d(A, B)=2 .
$$

From (i) and (ii), we obtain $u=\frac{x}{4}$ and $v=\frac{y}{4}$. Since $x \leq y$, it follows that $u \leq v$. Therefore

$$
\begin{aligned}
& \alpha((-1, u),(-1, v))=\frac{3}{4} \geq \frac{2}{3}=C_{\alpha}, \\
& \beta((-1, u),(-1, v))=\frac{1}{2} \leq \frac{1}{2}=C_{\beta} .
\end{aligned}
$$

Hence $T$ is an $\alpha-\beta$-proximal admissible.

We choose $x_{0}=(-1,1), x_{1}=\left(-1, \frac{1}{4}\right)$ in $A_{0}$. Then $x_{0} \preceq x_{1}$ and $d\left(\left(-1, \frac{1}{4}\right), T(-1,1)\right)=2=d(A, B)$. Also,

$$
\begin{aligned}
& \alpha\left((-1,1),\left(-1, \frac{1}{4}\right)\right)=\frac{3}{4} \geq \frac{2}{3}=C_{\alpha} \\
& \beta\left((-1,1),\left(-1, \frac{1}{4}\right)\right)=\frac{1}{2} \leq \frac{1}{2}=C_{\beta} .
\end{aligned}
$$

Hence all the hypotheses of Theorem 3.1 are satisfied and $(-1,0)$ and $(-1,4)$ are two best proximity points of $T$.

Here we observe that Condition $(H)$ of Theorem 3.3 fails to hold: if $u=\left(-1, x_{0}\right) \in A_{0}$ with $x_{0}>0$, we choose $x=(-1,0), y=(-1,4)$ so that $x=(-1,0) \npreceq\left(-1, x_{0}\right)=u$; if $u=(-1,0)$ then $x \preceq u$ and $y=$ $(-1,4) \preceq(-1,0)=u, \alpha(x, u)=\frac{3}{4}>\frac{2}{3}=C_{\alpha}, \beta(x, u)=\frac{1}{2}=C_{\beta}$ and $\beta(y, u)=0<\frac{1}{2}=C_{\beta}$. But $\alpha(y, u)=0 \nsupseteq \frac{2}{3}=C_{\alpha}$. 
Hence Condition $(H)$ of Theorem 3.3 fails to hold.

The following example is in support of Theorem 3.3 in which $T$ is not continuous.

Example 4.2. Let $X=[0,2] \times[0,2]$ with an Euclidean metric $d$.

We define a partial order on $X$ by $(x, y) \preceq(u, v)$ if and only if $x \geq u$ and $y \geq v$, for all $(x, y),(u, v) \in X$, where $\geq$ is the usual order on $\mathbb{R}$.

Let $A=\{0\} \times[0,2]=A_{0}, B=\{1\} \times[0,2]=B_{0}$.

We define $T: A \rightarrow B$ by

$$
T(0, x)= \begin{cases}\left(1, \frac{x^{2}}{1+2 x}\right), & \text { if } x \in[0,1], \\ \left(1, \frac{2}{3} x\right), & \text { if } x \in(1,2] .\end{cases}
$$

Clearly, $d(A, B)=1, T$ is not continuous, $T$ is proximally increasing on $A$ and $T\left(A_{0}\right) \subseteq B_{0}$.

Now, we define functions $\alpha, \beta: A \times A \rightarrow[0, \infty)$ by

$$
\begin{aligned}
& \alpha((0, x),(0, y))= \begin{cases}\frac{3}{4}, & \text { if } x, y \in[0,1] \text { with }(0, x) \preceq(0, y), \\
0, & \text { otherwise. }\end{cases} \\
& \beta((0, x),(0, y))= \begin{cases}\frac{2}{5}, & \text { if } x, y \in[0,1] \text { with }(0, x) \preceq(0, y), \\
0, & \text { otherwise. }\end{cases}
\end{aligned}
$$

Let $\psi(t)=\frac{7}{8} t$ for all $t \geq 0$. We now show that $T$ is an $\alpha-\beta-\psi$-proximal contractive mapping.

Now, let $(0, x),(0, y),(0, u)$ and $(0, v) \in A$ with $(0, x) \preceq(0, y)$ such that

$$
d((0, u), T(0, x))=d((0, v), T(0, y))=d(A, B)=1 .
$$

Let us consider the case $x, y \in[0,1]$ with $(0, x) \preceq(0, y)$. Then $\alpha((0, x)$, $(0, y))=\frac{3}{4}$ and $\beta((0, x),(0, y))=\frac{2}{5}$.

From (18), we obtain $u=\frac{x^{2}}{1+2 x} \in\left[0, \frac{1}{3}\right]$ and $v=\frac{y^{2}}{1+2 y} \in\left[0, \frac{1}{3}\right]$. Therefore

$$
\begin{aligned}
& \alpha((0, x),(0, y)) d((0, u),(0, v)) \\
= & \frac{3}{4}|u-v|=\frac{3}{4}\left|\frac{x^{2}}{1+2 x}-\frac{y^{2}}{1+2 y}\right| \\
= & \frac{3}{4}\left(\frac{|x-y|(x+y+2 x y) \mid}{(1+2 x)(1+2 y)}\right) \\
\leq & \frac{7}{20}|x-y|=\beta((0, x),(0, y)) \psi(d((0, x),(0, y))) .
\end{aligned}
$$

For the other possible cases the inequality (1) clearly holds. Hence $T$ is an $\alpha-\beta-\psi$-proximal contractive mapping.

We now show that $T$ is an $\alpha-\beta$-proximal admissible. For this purpose, we choose $C_{\alpha}=\frac{2}{3}$ and $C_{\beta}=\frac{1}{2}$. Clearly $0 \leq \frac{C_{\beta}}{C_{\alpha}} \leq 1$.

Let $(0, x),(0, y),(0, u)$ and $(0, v) \in A$ with $x \geq y$ such that 
(a) $\alpha((0, x),(0, y))=\frac{3}{4} \geq \frac{2}{3}=C_{\alpha}$, $d((0, u), T(0, x))=d((0, v), T(0, y))=d(A, B)=1$,

(b) $\beta((0, x),(0, y))=\frac{2}{5} \leq \frac{1}{2}=C_{\beta}$, $d((0, u), T(0, x))=d((0, v), T(0, y))=d(A, B)=1$.

From $(a)$ and $(b)$, we obtain $u=\frac{x^{2}}{1+2 x} \in\left[0, \frac{1}{3}\right]$ and $v=\frac{y^{2}}{1+2 y} \in\left[0, \frac{1}{3}\right]$.

Since $x \geq y$, it follows that $u \geq v$. Therefore $\alpha((0, u),(0, v))=\frac{3}{4} \geq \frac{2}{3}=$ $C_{\alpha}$ and $\beta((0, u),(0, v))=\frac{2}{5} \leq \frac{1}{2}=C_{\beta}$.

Hence $T$ is an $\alpha-\beta$-proximal admissible.

Now, we choose $x_{0}=\left(0, \frac{1}{2}\right), x_{1}=\left(0, \frac{1}{8}\right)$, such that $d\left(\left(0, \frac{1}{8}\right), T\left(0, \frac{1}{2}\right)\right)=1$, $\alpha\left(\left(0, \frac{1}{2}\right),\left(0, \frac{1}{8}\right)\right)=\frac{3}{4} \geq \frac{2}{3}=C_{\alpha}, \beta\left(\left(0, \frac{1}{2}\right),\left(0, \frac{1}{8}\right)\right)=\frac{2}{5} \leq \frac{1}{2}=C_{\beta}$ and $x_{0} \preceq x_{1}$.

Finally, if $\left\{x_{n}\right\}$ is a sequence in $A$ such that $\alpha\left(x_{n}, x_{n+1}\right) \geq C_{\alpha}, \beta\left(x_{n}, x_{n+1}\right)$ $\leq C_{\beta}$ for all $n \in \mathbb{N}$ and $x_{n} \rightarrow x$ as $n \rightarrow \infty$, then by definition of $\alpha$ and $\beta$, $x_{n} \in[0,1]$. Thus $x \in[0,1]$. i.e., $\alpha\left(x_{n}, x\right) \geq C_{\alpha}$ and $\beta\left(x_{n}, x\right) \leq C_{\beta}$. Further, $\left\{x_{n}\right\}$ is a nondecreasing sequence, then $x_{n} \preceq x$ for all $n \in \mathbb{N}$.

Hence all the hypotheses of Theorem 3.3 are satisfied and $(0,0)$ is the unique best proximity point of $T$.

\section{REFERENCES}

[1] A. Abkar, M. Gabeleh, Best proximity points of non-self mappings, Top, 21 (2) (2013), 287-295.

[2] A. Abkar, M. Gabeleh, Global optimal solutions of noncyclic mappings in metric spaces, J. Optim. Theory Appl., 153 (2) (2012), 298-305.

[3] M. A. Al-Thaga, N. Shahzad, Convergence and existence results for best proximity points, Nonlinear Anal., 70 (10) (2009), 3665-3671.

[4] M. S. Asgari and Z. Badehian, Fixed Point theorems for $\alpha-\beta-\psi-$ Contractive Mappings in partially Ordered sets, J. Nonlinear Sci. and Appl., 8 (2015), 518-528.

[5] S. S. Basha, Best proximity points, optimal solutions, J. Optim. Theory Appl., 151 (2011), 210-216.

[6] S. S. Basha, Discrete optimization in partially ordered sets, J. Glob. Optim., 54 (3) (2012), 511-517.

[7] J. Caballero, J. Harjani, K. Sadarangani, A best proximity point theorem for Geraghty-contractions, Fixed Point Theory Appl., 2012, Article ID 231, (2012), 9 pages.

[8] B. S. Choudhury, N. Metiya, M. Postolache and P. Konar, A discussion on best proximity point and coupled best proximity point in partially ordered metric space, Fixed Point Theory Appl., 2015 (2015), Article ID 170, 17 pages.

[9] B. S. Choudhury, P. Maity, P. Konar, A global optimality result using nonself mappings, Opsearch, 51 (2) (2014), 312-320.

[10] B. S. Choudhury, P. Maity, P. Konar, A global optimality result using Geraghty type contraction, Int. J. Optim. Control, Theor. Appl., 4(2) (2014), 99-104.

[11] A. A. Eldred, P. Veeramani, Existence and convergence of best proximity points, J. Math. Anal. Appl., 323 (2) (2006), 1001-1006. 
[12] K. Fan, Extensions of two fixed point theorems of F.E. Browder, Math. Z., 112 (3) (1969), 234-240.

[13] M. Gabeleh, Best proximity point theorems via proximal non-self mappings, J. Optim. Theory Appl., 164 (2) (2015), 565-576.

[14] J. Hamzehnejadi and R. Lashkaripour, Best proximity points for generalized $\alpha-\phi-$ Geraghty proximal contraction mappings and its application, Fixed point theory Appl., 2016 (2016), Article ID 72, 13 pages.

[15] E. Karapinar, On best proximity point of $\psi$-Geraghty contractions, Fixed Point Theory Appl., 2013 (2013), Article ID 200, 9 pages.

[16] M. A. Kutbi, S. Chandok, W. Sintunavarat, Optimal solutions for nonlinear proximal CN-contraction mapping in metric space, J. Inequal. Appl., 2014 (2014), Article ID 193, 9 pages.

[17] V. Sankar Raj, A best proximity point theorem for weakly contractive non-self mappings, Nonlinear Anal., 74 (14) (2011), 4804-4808.

Gutti Venkata Ravindranadh Babu

Department of Mathematics

ANDHRA UNIVERSITY

VISAKHAPATNAM-530 003

INDIA

E-mail address: gvr_babu@hotmail.com

Leta Bekere Kumssa

Department of Mathematics

Madda Walabu University

Bale RoBe

P.O. Box 247

ETHIOPIA

Department of Mathematics

ANDHRA UNIVERSITY

VISAKHAPATNAM-530 003

INDIA

E-mail address: letabekere@yahoo.com

Pathina Sudheer Kumar

Department of Mathematics

ANDHRA UNIVERSITY

VISAKHAPATNAM-530 003

INDIA

E-mail address: sudheer232.maths@hotmail.com 